\title{
Classification and quality of groundwater supplies in the Lower Shire Valley, Malawi - Part 1: Physico-chemical quality of borehole water supplies in Chikhwawa, Malawi
}

\author{
AM Grimason ${ }^{1,2,4^{*}}$, TD Morse ${ }^{1,2,3,4}$, TK Beattie ${ }^{3}$, SJ Masangwi' ${ }^{2}$, GC Jabu ${ }^{1,2}$, SC Taulo ${ }^{1,2}$ and KK Lungu ${ }^{1,2,4}$ \\ 'Department of Environmental Health, University of Malawi - The Polytechnic, P/B 303 Chichiri, Blantyre, Malawi \\ ${ }^{2}$ Centre for Water, Sanitation, Health and Appropriate Technology Development (WASHTED), University of Malawi - \\ The Polytechnic, P/B 303 Chichiri, Blantyre, Malawi \\ ${ }^{3}$ Environmental Health, Department of Civil Engineering, University of Strathclyde, Glasgow, U.K. G1 ONG \\ ${ }^{4}$ Africa Academy for Environmental Health, PO Box 15574, Sinoville 0129, South Africa
}

\begin{abstract}
This paper presents data on the physico-chemical quality of groundwater supplies in Chikhwawa, Malawi. Eighty-four water samples were collected and analysed for a range of chemical constituents ( $\mathrm{Al}, \mathrm{As}, \mathrm{Ca}, \mathrm{Cd}, \mathrm{Co}, \mathrm{Cr}, \mathrm{Cu}, \mathrm{Fe}, \mathrm{Hg}, \mathrm{Mg}, \mathrm{Mn}, \mathrm{Ni}$, $\left.\mathrm{Pb}, \mathrm{Se}, \mathrm{V}, \mathrm{Zn}, \mathrm{K}, \mathrm{Na}, \mathrm{Cl}, \mathrm{F}-\mathrm{NO}_{3}, \mathrm{SO}_{4}^{2-}\right)$, $\mathrm{pH}$, temperature, electrical conductivity and turbidity, from 28 boreholes located in 25 remote, rural villages ( $n=3$ per village) distributed along the east $(n=15)$ and west $(n=10)$ banks of the Shire River. Samples were collected every 2 months during the wet season, over a period of 5 months (December to April). Results were compared with national (Malawi Bureau of Standards Maximum Permissible Levels (MBS MPL)) and international (World Health Organization Guideline Values (WHO GV)) drinking-water standards. In general, most parameters complied with the Malawi Bureau of Standards Maximum Permissible Levels (MBS MPL) for borehole water supplies. The MBS MPL standards for iron, sodium and nitrate were slightly exceed at a few boreholes, technically rendering the water supply unwholesome but not necessarily unfit for human consumption. In contrast, significantly high nitrate $(<200 \mathrm{mg} / \ell)$ and fluoride $(\leq 5 \mathrm{mg} / \ell)$ concentrations at levels which constitute a significant risk to the health of the consumer were detected in borehole samples in a number of villages and warrant further investigation. Water committee members complained of problems associated with taste (saltiness or bitterness) and appearance (discoloured water) primarily on the west bank, presumably as a result of the high sodium and chloride levels, and precipitation of soluble iron and manganese, respectively. This resulted in some water collectors reverting to the use of surface water sources to obtain drinking-water, a practice which should be dissuaded through the education of water and village health committees.
\end{abstract}

Keywords: Wholesomeness, fitness for human consumption, borehole water quality, Malawi

\section{INTRODUCTION}

The chemical quality of groundwater used for human consumption in developing countries is a significant, but neglected public health issue. In Malawi, like many countries in Africa, abstracted groundwater derived from a 'protected or improved' source is often the main source of 'safe' drinking-water for the poor in rural areas. The fact that water is abstracted from underground and from an 'improved' source is often assumed by the local community to mean that the water is 'safe' for human consumption. However, this is an over-simplification and it should not be automatically assumed that such water is indeed 'safe' for human consumption.

To determine the wholesomeness of water supplies in the developed world, water samples are routinely taken and measured against a range of health and non-health based physicochemical standards and bacteriological indicators of faecal pollution. The majority of these standards are based on the WHO Health-Based Guidelines Values, which have been adapted and/

To whom all correspondence should be addressed.

푤 00265992303140; e-mail: agrimason@poly.ac.mw or tonygrimason@yahoo.co.uk

Received 13 December 2012; accepted in revised form 4 July 2013. or amended by governments to derive appropriate National Standards. In Malawi, for borehole water quality to satisfy the requirements of wholesomeness it must be safe from waterborne or associated disease, free from toxic chemicals and be aesthetically and organoleptically acceptable to the consumer (MBS, 2005). In addition, compliance is also required with respect to Malawi Bureau of Standards (MBS) derived maximum permissible levels (MPLs) for 17 chemicals in drinkingwater and 11 physical and macro-constituent characteristics.

Monitoring of drinking-water quality is an expensive exercise and is usually only undertaken on a regular basis by statutory suppliers of drinking-water to large conurbations. Regular monitoring of small community water supplies such as boreholes in remote, rural areas of developing countries is unrealistic. Although monitoring is important, it should not be undertaken at the expense of maximising the provision of additional 'safe' sources of water supplies to communities that access their drinking-water from unprotected sources (WHO, 2010).

In Malawi, the Ministry of Irrigation and Water Development recommends that upon construction of a borehole, and before maintenance and control is officially handed over to the community, a small number of water quality tests are undertaken, i.e., $\mathrm{pH}$, total dissolved solids, sulphates, nitrates, fluoride, chloride, electrical conductivity and iron 


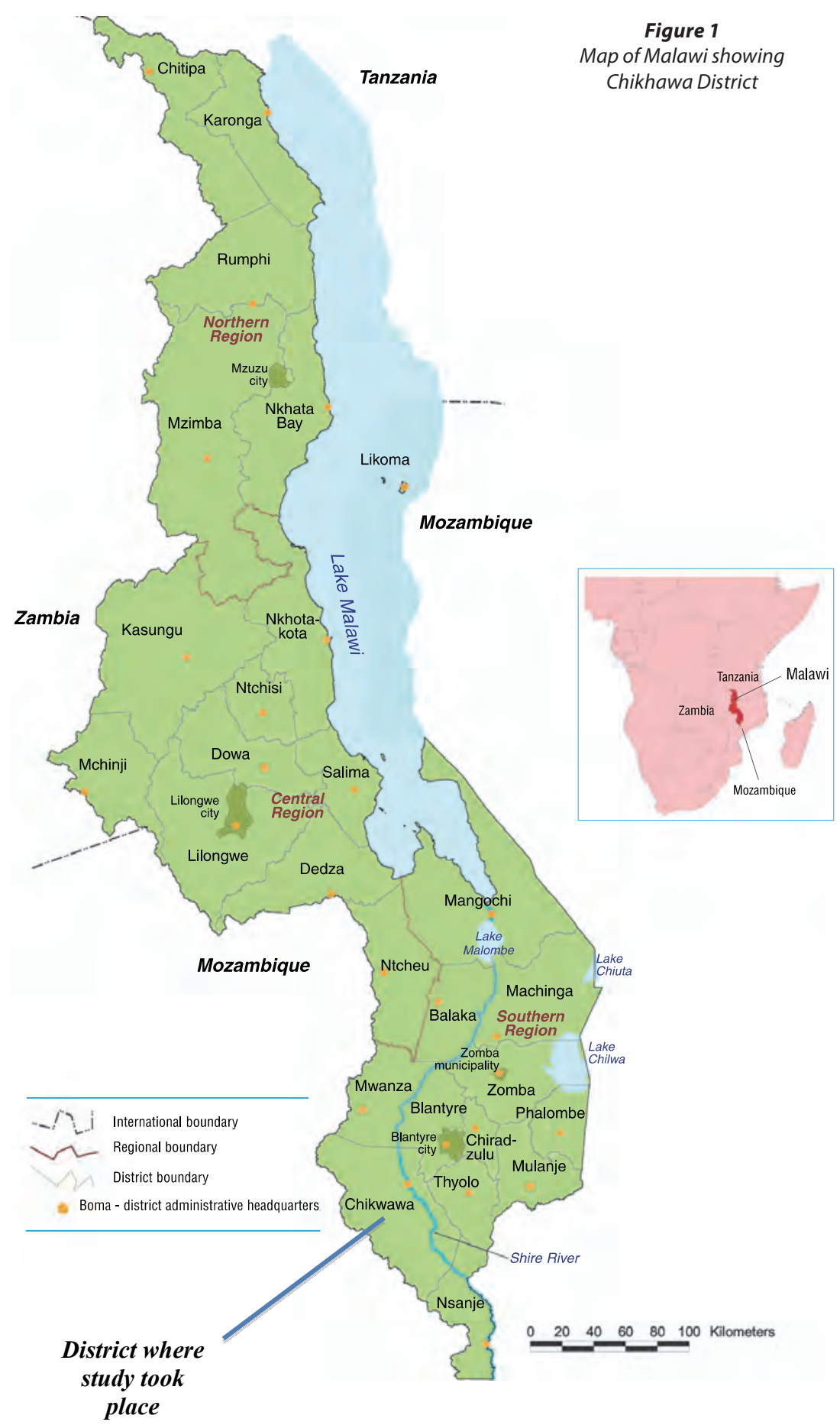

people obtain their drinking-water from borehole supplies (NSO, 2005). At the present time it is estimated that there are 997 (806 functional) boreholes in Chikhwawa (Nchilikiso, 2011).

Despite the vast range of health-based guideline values recommended by the WHO for chemicals in drinking-water, only a few chemicals have actually been shown to cause widespread health effects in humans as a consequence of exposure through drinking-water and when present in excessive quantities, i.e. fluoride, arsenic and nitrate (WHO, 2008). Although borehole supplies are clearly used by a significant percentage of the population in Malawi, limited information exists on the chemical composition of borehole water supplies in Chikhwawa (Bath, 1980; Chilton and Smith-Carington, 1984; UN, 1989; Sajidu et al., 2008). To this end, a study was undertaken to determine the acceptability, wholesomeness and fitness for human consumption of borehole water supplies in Chikhwawa, Malawi, based upon national and international drinkingwater standards.

\section{STUDY AREA}

Malawi is a small landlocked southeastern African country, approximately $900 \mathrm{~km}$ long and $80-250 \mathrm{~km}$ wide, with an area of about $120000 \mathrm{~km}^{2}$ (Fig. 1). Chikhwawa is located in the Lower Shire Valley, on the southern tip of the East African Rift Valley system $\left(16^{\circ} 00^{\prime} \mathrm{S}\right.$ $34^{\circ} 50^{\prime} \mathrm{E}$ ). The Lower Shire covers a total of $6730 \mathrm{~km}^{2}$, although the valley floor is slightly less than $2590 \mathrm{~km}^{2}$. The area is low-lying, at about $100 \mathrm{~m}$ amsl. The Thyolo Escarpment forms the boundary on the east of the valley and on the west there is the international boundary with Mozambique, which also forms the surface water divide between the Shire and Zambezi catchments. The Shire River splits Lower Shire into two distinctive areas known as the east and west banks (Fig. 1).
(MIWD, 2002). In reality, this is seldom undertaken by both Government and non-governmental organisation providers of borehole supplies (Kanyerere et al., 2010).

Out of the 27 districts in Malawi, 23 (85\%) depend on groundwater as the main source of water supply to people living in rural areas (Kanyerere et al., 2010). In 2004 the National Statistics Office (NSO) of Malawi reported that nearly half (46.5\%) of the total population in Malawi access their drinking-water from borehole supplies (NSO, 2005). In 2007, the results of a national water point mapping survey revealed that 35000 groundwater sources were being used by rural populations for drinking-water purposes (Kanyerere et al., 2010). In Chikhwawa, the NSO of Malawi reported that 1 in 2

\section{METHODOLOGY}

Eighty-four groundwater samples were collected for chemical analyses from boreholes $(n=28)$ located in 25 remote, rural villages in the Chikhwawa District ( $n=3$ per borehole) distributed along the east $(n=15)$ and west $(n=10)$ banks of the River Shire. Boreholes (BH) were located in the Traditional Authority areas of Mulilima (Villages 1 and 2), Katunga (BH 3-8), Maseya (BH 9-11), Mulilima (BH 12-16) on the east bank of the river, and Katunga (17-19), Kasisi (BH 20) and Katunga (BH 21-28) on the west bank of the river (Fig. 2).

Samples were collected every 2 months during the wet season (December, February and April) in 2001/02. Samples were 


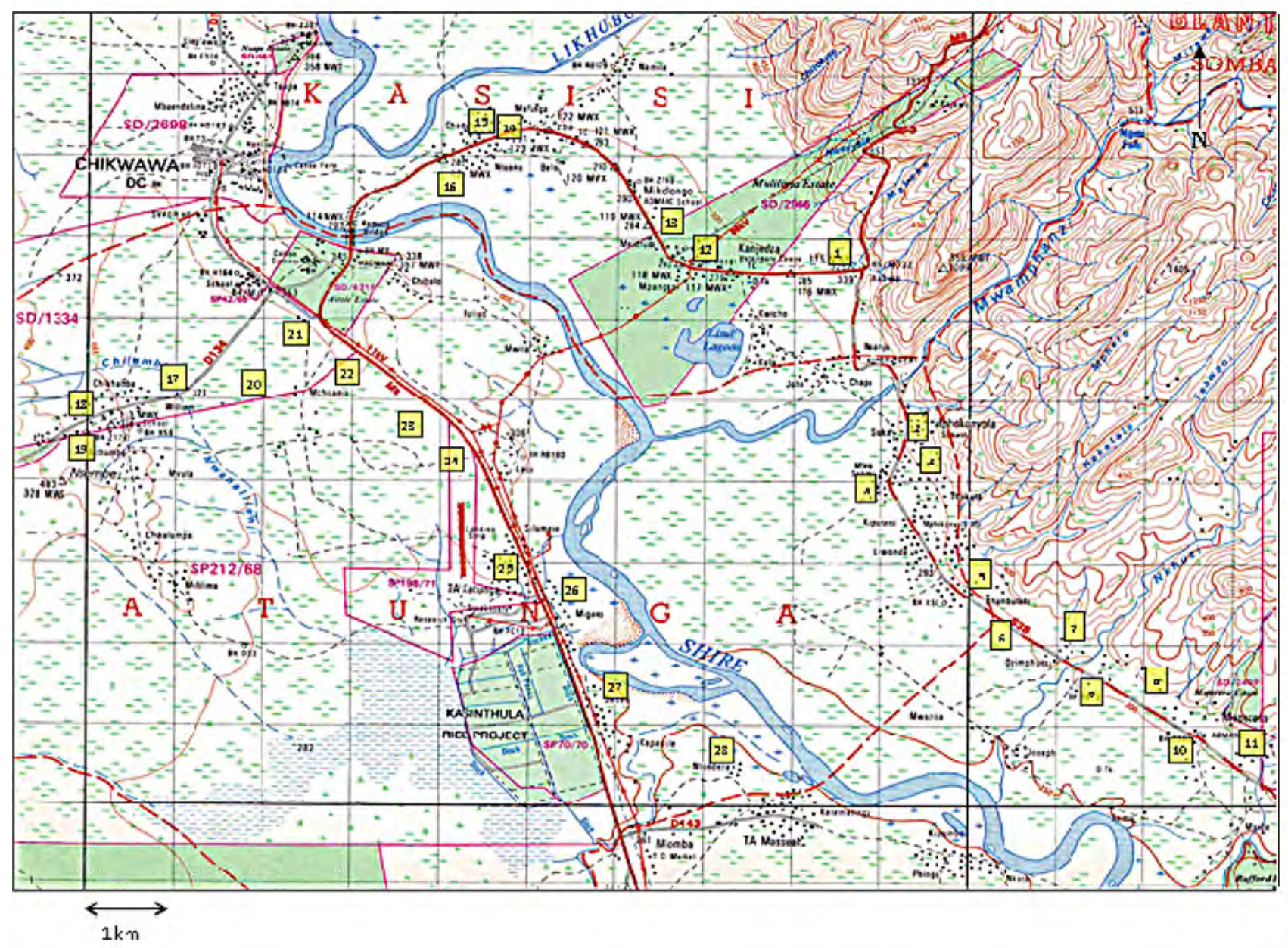

Figure 2

Map of study area outlining location of borehole water supplies sampled on the east and west banks of the Shire River, Chikhwawa

collected directly from the borehole without pumping water to waste. Five hundred millilitre $(500 \mathrm{~m} \ell)$ samples of water from each borehole were collected in sterile polypropylene bottles and analysed for the following chemical constituents $\left(\mathrm{Cl}^{-}, \mathrm{F}^{-}\right.$, $\mathrm{NO}_{3}, \mathrm{SO}_{4}{ }^{2}, \mathrm{Al}, \mathrm{As}, \mathrm{Ca}, \mathrm{Cd}, \mathrm{Co}, \mathrm{Cr}, \mathrm{Cu}, \mathrm{Fe}, \mathrm{Hg}, \mathrm{Mg}, \mathrm{Mn}, \mathrm{Ni}, \mathrm{Pb}$, $\mathrm{Se}, \mathrm{V}, \mathrm{Zn}, \mathrm{Na}, \mathrm{K})$. Each sample was filtered through a $0.45 \mu \mathrm{m}$ polytetrafluoroethlyene (PTFE) syringe filter (13 $\mathrm{mm}$ dia.).

Filtrates for cations were transferred into sterile $50 \mathrm{ml}$ polypropylene centrifuge tubes, acidified to $\mathrm{pH}<2$ by the addition of Merck ultra-pure nitric acid and stored at $4^{\circ} \mathrm{C}$ until analysed (7 to 30 days post-collection). Samples for anions were transferred into sterile $50 \mathrm{~m} \ell$ polypropylene centrifuge tubes and similarly stored at $4^{\circ} \mathrm{C}$ without preservation ( 7 to 30 days post collection). Anions were detected using the DIONEX 2000i ion chromatography system and cations using a sequential inductively coupled plasma atomic emission spectrometer (ICP-AES) (Jobin Yvonne JY-24) at the Department of Civil Engineering, University of Strathclyde, Glasgow. All analyses were carried out in accordance with standard methods for the examination of water and wastewater (APHA, 1998).

Temperature, $\mathrm{pH}$, electrical conductivity and turbidity were measured in situ using portable HANNA meters. All equipment was calibrated on a daily basis using standard solutions. Analysis was conducted as soon as water samples were collected. To address issues such as taste, appearance, colour and acceptability of borehole water, various members of the water committee in each village were consulted. The water committee are a voluntary group of individuals who are trained in the maintenance and management of the borehole within their community to ensure that it is running efficiently and effectively. The committee also play a role in the education of the community on drinking water safety, hygiene and sanitation in the community.

Results are presented in the form of the range ( $\min -\max$ ), mean \pm standard deviation (SD) and median, and have been compared with national (Malawi Bureau of Standards Maximum Permissible Levels) and international (World Health Organization Health-Based Guidelines Values) standards (Table 1).

\section{RESULTS AND DISCUSSION}

\section{Age, location and geographical terrain of boreholes}

As shown in Table 2 the majority (75\%) of the borehole water supplies tested had been constructed within 4 years of the study period; 2 boreholes were about a decade old and the remaining 4 boreholes constructed between 25 and 35 years ago. Very few water committee members could remember the depth of their borehole, so no comment can be offered as to whether the aquifers under investigation were shallow or deep. Boreholes were distributed along the east $(n=15)$ and west $(n=10)$ banks of the river Shire (Fig. 2). 


\begin{tabular}{|l|l|l|}
\hline \multicolumn{3}{|c|}{$\begin{array}{c}\text { TABLE } 1 \\
\text { Select Malawi Bureau of Standards Maximum Permissible } \\
\text { Levels (MBS, 2005) and World Health Organization Drinking- } \\
\text { Water Guideline Values (WHO, 2008) }\end{array}$} \\
\hline Parameter & MBS MPL & WHO GV \\
\hline Turbidity & $25 \mathrm{NTU}$ & Guideline value not stated \\
\hline $\begin{array}{l}\text { Electrical } \\
\text { conductivity }\end{array}$ & $3500 \mu \mathrm{S} / \mathrm{cm}$ & Guideline value not stated \\
\hline pH value & $6-9$ & Guideline value not stated \\
\hline Copper & $2 \mathrm{mg} / \ell$ & $2 \mathrm{mg} / \ell$ \\
\hline Magnesium & $200 \mathrm{mg} / \ell$ & Guideline value not stated \\
\hline Iron & $3 \mathrm{mg} / \ell$ & No guideline value proposed \\
\hline Zinc & $15 \mathrm{mg} / \ell$ & No guideline value proposed \\
\hline Manganese & $1.5 \mathrm{mg} / \ell$ & $0.4 \mathrm{mg} / \ell$ \\
\hline Chromium & $10 \mu \mathrm{g} / \ell$ & $50 \mu \mathrm{l} / \ell$ \\
\hline Sodium & $500 \mathrm{mg} / \ell$ & No guideline value proposed $^{*}$ \\
\hline Calcium & $250 \mathrm{mg} / \ell$ & Guideline value not stated \\
\hline Chloride & $750 \mathrm{mg} / \ell$ & No guideline value proposed \\
\hline Fluoride & $6.0 \mathrm{mg} / \ell$ & $1.5 \mathrm{mg} / \ell$ \\
\hline Nitrate & $45 \mathrm{mg} / \ell$ & $50 \mathrm{mg} / \ell$ \\
\hline Sulphate & $800 \mathrm{mg} / \ell$ & Guideline value not stated ${ }^{*}$ \\
\hline $\begin{array}{l}\text { Faecal } \\
\text { coliforms }\end{array}$ & $\begin{array}{l}50 \mathrm{cfu} / \\
100 \mathrm{~m} \ell\end{array}$ & Guideline value not stated ${ }^{*}$ \\
\hline $\begin{array}{l}\text { Faecal } \\
\text { enterococci }\end{array}$ & $\begin{array}{l}0 \mathrm{cfu} / \\
100 \mathrm{~m} \ell\end{array}$ & Guideline value not stated \\
\hline & \\
\hline
\end{tabular}

${ }^{*}$ Whilst the $1^{\text {st }}$ and $2^{\text {nd }}$ editions of the WHO Guidelines for DrinkingWater Quality recommend various guideline values for a number of the parameters outlined above, the $3^{\text {rd }}$ and $4^{\text {th }}$ (WHO, 2011) editions of the WHO Guidelines for Drinking-Water Quality do not.

\section{Physical characteristics of borehole water samples}

The physical parameters and results of the chemical concentrations detected for each borehole water sample are presented in Tables 2-4. Little difference was observed between the range of $\mathrm{pH}(6.8-7.9)$ and temperature $\left(28.1-31.5^{\circ} \mathrm{C}\right)$ of water samples recorded in situ from both banks of the Shire River. The range of $\mathrm{pH}$ values recorded comply with the Malawi Standards (MBS) maximum permissible levels (MPL), i.e., range 6.0-9.5, for borehole water supplies (MBS, 2005). The $\mathrm{pH}$ and temperatures recorded are in accordance with those expected for borehole water supplies in Malawi (Chilton and Smith-Carington, 1984; McFarlane and Bowden, 1992; Palamuleni, 2002; Sajidu et al., 2008) and Africa (Langenegge, 1994; Bordalo and SavvaBordalo, 2007).

Electrical conductivity (EC) is a valuable indicator of the minerals dissolved in water. Marked differences were observed in EC values from borehole water samples on the east $(<1000$ $\mu \mathrm{S} / \mathrm{cm})$ and west bank $(1000-7000 \mu \mathrm{S} / \mathrm{cm})$ of the Shire River. Generally, groundwater on the east bank had relatively low conductivity values compared with the significantly higher levels recorded on the west bank. Whereas all waters samples on the east bank of the river and 9 of the 12 boreholes on the west bank complied with the MBS MPL, samples collected from Lakiuji, Migano and Mtondeza on the west bank exceeded the MPL on each occasion.

Interestingly, these three boreholes also had the highest levels of sodium recorded and were amongst the top four highest concentrations recorded for potassium, chloride, fluoride and sulphate. Elevated concentrations of calcium and magnesium were also recorded in these water samples. These results are in accordance with those of Bath (1980) who reported particularly high levels of sodium and chloride in groundwaters from the Lower Shire. Two of these boreholes were the subject of consumer complaints associated with the 'saltiness' of the water. Earlier studies in Malawi reported wide ranges in the electrical conductance values in water samples from boreholes in Nkhotakota (180-4 $600 \mu \mathrm{S} / \mathrm{cm})$, South Rukuru (70-7 $700 \mu \mathrm{S} /$ $\mathrm{cm})$, Livulezi and Dowa West $(<750-4000 \mu \mathrm{S} / \mathrm{cm})$ (Bath, 1980; Chilton and Smith-Carington, 1984), although the majority of samples in Livulezi and Dowa West were below $750 \mu \mathrm{S} / \mathrm{cm}$ ). Sajidu et al., (2008) reported similar wide ranging EC values for borehole water supplies in Chikhwawa (1 450-2 $800 \mu \mathrm{S} / \mathrm{cm})$, Nsanje (2 150-6 600 $\mu \mathrm{S} / \mathrm{cm})$, Mangochi (295-6 $800 \mu \mathrm{S} / \mathrm{cm})$, Zomba $(129-805 \mu \mathrm{S} / \mathrm{cm})$ and Machinga (55-1 $175 \mu \mathrm{S} / \mathrm{cm})$.

Turbidity levels ranged from 0 to 5.9 NTU (median 2 NTU) in water samples from the east bank and 0 to $5.6 \mathrm{NTU}$ (median $0.6 \mathrm{NTU}$ ) on the west bank; all were well below the MBSMPL. The majority (93\%) of samples were below 2.5 NTU, with the exception of water samples from Dyelatu School and Migano. Whilst turbidity does not have a direct effect on the health of the consumer, turbid water may be unacceptable to consumers from an aesthetic viewpoint. The threshold at which turbidity can be detected by the naked eye is 5 NTU (WHO, 2008). Most studies conducted in Africa (including Malawi) on groundwater supplies have reported turbidity values of less than $1 \mathrm{NTU}$; however values greater than 10 NTU and up to 50 NTU have been recorded (Langenegge, 1994; Bordalo and Savva-Bordalo, 2007; Sajidu et al., 2007).

Levels of calcium, potassium, sodium, sulphates and chlorides at Dyelatu School and Migano were amongst the highest recorded. These ions are known to influence iron corrosion (WHO, 2008). High turbidity levels may also be due to cement deterioration, which can occur on prolonged exposure to aggressive water containing elevated chloride and sulphate concentrations (WHO, 2008). This can cause the groundwater to become contaminated through the presence of suspended corrosion products, and mud entering wells because of defective aprons caused by poor connectivity between the handpump and the apron or improperly installed pumps (Langenegge, 1994).

\section{Chemical constituents of borehole water samples}

Nitrate concentrations in borehole supplies were highly variable on both banks of the river. With the exception of samples collected from Khumbulani on the east bank and Wiliyamu I and Migano on the west bank, all borehole samples complied with the MBS MPL. The MBS MPL for nitrate is similar to the WHO health-based GV which is aimed at protecting infants. Fifty per cent of samples collected from both banks contained low to medium nitrate concentrations of up to $<10 \mathrm{mg} / \ell$, one in three had concentrations between 10 and $40 \mathrm{mg} / \ell$ and $14 \%$ had concentrations $>40 \mathrm{mg} / \ell$ : Mfela School and Khumbulani on the east bank and Wiliyamu I and Migano on the west bank. Nitrate levels in groundwater at Migano were, on average, 4 times the recommended MBS MPL.

Studies conducted of groundwater quality in the Lower Shire, Nkhotakota, Bua and South Rukuru over 20 years ago revealed that the majority of samples had nitrate levels $<1 \mathrm{mg} / \ell$ (Bath, 1980; UN 1989). However, sporadic high concentrations were detected from time to time at levels up to $41 \mathrm{mg} / \ell, 6.1$ $\mathrm{mg} / \ell, 6.3 \mathrm{mg} / \ell$ and $9.3 \mathrm{mg} / \ell$, respectively (Bath, 1980). More recently, a study by Sajidu et al. (2007) conducted in Machinga 


\begin{tabular}{|c|c|c|c|c|c|c|c|c|}
\hline \multicolumn{9}{|c|}{$\begin{array}{l}\text { TABLE } 2 \\
\text { Physical parameters of borehole water samples collected from the east (BH 1-16) and west (BH 17-28) bank of the Shire River }\end{array}$} \\
\hline $\begin{array}{l}\text { BH } \\
\text { No. }\end{array}$ & Village/Area & \begin{tabular}{|c|}
$\begin{array}{c}\text { Age of } \\
\text { borehole } \\
\text { (Years) }\end{array}$ \\
\end{tabular} & Provider & $\begin{array}{c}\text { Pop. } \\
\text { supplied }\end{array}$ & $\mathrm{pH}$ & $\begin{array}{l}\text { Temperature } \\
\left({ }^{\circ} \mathrm{C}\right)\end{array}$ & $\begin{array}{c}\text { Electrical } \\
\text { conductivity } \\
(\mu \mathrm{S} / \mathrm{cm})\end{array}$ & $\begin{array}{l}\text { Turbidity } \\
\text { (NTU) }\end{array}$ \\
\hline 1 & Mkhwicho & 4 & MIWD & 500 & $7.3 \pm 0.1$ & $28.7 \pm 0.6$ & $591.0 \pm 44.2$ & $2.1 \pm 0.1$ \\
\hline 2 & Matumula & 1 & MASAF & 500 & $7.4 \pm 0.1$ & $29.1 \pm 0.1$ & $602.3 \pm 90.9$ & $2.5 \pm 0.1$ \\
\hline 3 & Mpokonyola & 3 & MASAF & 250 & $7.5 \pm 0.1$ & $29.3 \pm 0.1$ & $346.3 \pm 84.5$ & $2.2 \pm 0.1$ \\
\hline 4 & Mfela School & 35 & CCAP & 1000 & $7.4 \pm 0.2$ & $29.0 \pm 0.9$ & $660.7 \pm 56.6$ & $2.1 \pm 0.1$ \\
\hline 5 & Kapufeni & 3 & MIWD & 250 & $7.4 \pm 0.2$ & $29.6 \pm 0.8$ & $325.3 \pm 48.8$ & $1.8 \pm 0$ \\
\hline 6 & Khumbulani & 9 & MIWD & 400 & $7.3 \pm 0.1$ & $30.0 \pm 0.9$ & $634.7 \pm 148.0$ & $1.9 \pm 0$ \\
\hline 7 & Chinkole & 3 & MASAF & 500 & $7.4 \pm 0.1$ & $30.8 \pm 0.5$ & $959.3 \pm 74.8$ & $2.0 \pm 0.1$ \\
\hline 8 & Khumbulani & 3 & MASAF & 1,000 & $7.4 \pm 0.2$ & $30.3 \pm 0.6$ & $431.0 \pm 62.2$ & $1.6 \pm 0.5$ \\
\hline 9 & Zimphutsi & 1 & UNICEF & 450 & $7.2 \pm 0.1$ & $29.3 \pm 1.1$ & $865.3 \pm 86.6$ & $1.6 \pm 1.1$ \\
\hline 10 & Samu & 2 & MASAF & 250 & $6.9 \pm 0.1$ & $29.6 \pm 0.5$ & $860.7 \pm 3.2$ & $1.6 \pm 0.7$ \\
\hline 11 & Admarc Market (AM) & 32 & AM \&MIWD & 200 & $7.2 \pm 0.0$ & $29.0 \pm 0.8$ & $887.3 \pm 20.6$ & $1.5 \pm 0$ \\
\hline 12 & Mpangeni & 25 & CCAP & 400 & $7.4 \pm 0.2$ & $29.9 \pm 0.3$ & $893.0 \pm 163.1$ & $1.8 \pm 0.1$ \\
\hline 13 & Bello & 9 & MIWD & 250 & $7.6 \pm 0.2$ & $28.5 \pm 0.8$ & $486.0 \pm 83.7$ & $1.0 \pm 0$ \\
\hline 14 & Chadula I & 1 & MASAF & 500 & $7.5 \pm 0.2$ & $28.2 \pm 0.2$ & $220.7 \pm 115.8$ & $1.1 \pm 0$ \\
\hline 15 & Chadula II & 1 & MASAF & 500 & $7.6 \pm 0.2$ & $28.7 \pm 0.8$ & $364.7 \pm 17.0$ & $1.1 \pm 0.7$ \\
\hline 16 & Mtuwawa & 26 & SCF & 500 & $7.5 \pm 0.3$ & $28.8 \pm 0.6$ & $320.0 \pm 100.9$ & $1.0 \pm 0.1$ \\
\hline 17 & Wiliyamu I & 2 & PROSCAP & 30 & $7.1 \pm 0.2$ & $30.0 \pm 0.8$ & $1206 \pm 173.3$ & $2.2 \pm 0.6$ \\
\hline 18 & Wiliyamu II & $\mathrm{nk}$ & $\mathrm{Nk}$ & 500 & $7.4 \pm 0.4$ & $28.9 \pm 0.8$ & $1341.3 \pm 75.7$ & 0.0 \\
\hline 19 & Nedi & 1 & MASAF & 600 & $7.3 \pm 0.1$ & $30.2 \pm 0.8$ & $2164.0 \pm 218.2$ & $0.1 \pm 0.1$ \\
\hline 20 & Kanthema & 2 & MASAF & 500 & $7.3 \pm 0.1$ & $30.1 \pm 0.3$ & $1823.7 \pm 25.4$ & 0.0 \\
\hline 21 & Kabudula I & 2 & MASAF & 750 & $7.6 \pm 0.2$ & $30.8 \pm 0.6$ & $1367.7 \pm 49.7$ & $0.5 \pm 0.6$ \\
\hline 22 & Kabudula II & 2 & MASAF & 150 & $7.6 \pm 0.2$ & $30.7 \pm 0.1$ & $1967.0 \pm 55.4$ & 0.0 \\
\hline 23 & Lawyi 2 & 2 & WB & 250 & $7.7 \pm 0.1$ & $30.7 \pm 0.6$ & $1826.3 \pm 62.9$ & $1.2 \pm 0.3$ \\
\hline 24 & Dyelatu School & 4 & MASAF & 1000 & $7.5 \pm 0.2$ & $30.0 \pm 0.9$ & $2454.3 \pm 94.1$ & $3.9 \pm 0.5$ \\
\hline 25 & Lakiuji & 2 & MASAF & 50 & $7.4 \pm 0.1$ & $30.3 \pm 0.4$ & $4307.7 \pm 186.5$ & $2.2 \pm 0.3$ \\
\hline 26 & Migano & 2 & MASAF & 500 & $7.3 \pm 0.1$ & $30.7 \pm 0.5$ & $4055.0 \pm 77.9$ & $5.6 \pm 0$ \\
\hline 27 & Sisev & 2 & MASAF & 200 & $7.6 \pm 0.3$ & $29.2 \pm 0.3$ & $1053.3 \pm 75.1$ & 0.0 \\
\hline 28 & Mtondeza & 2 & MASAF & 200 & $7.5 \pm 0.2$ & $30.7 \pm 0.2$ & $6574.3 \pm 180.7$ & 0.0 \\
\hline
\end{tabular}

$B H=$ Borehole Number; MIWD = Ministry of Irrigation \& Water Development UNICEF = United Nations

Children's Fund; $C C A P=$ Church of Central Africa Presbyterian; $S C F=$ Save the Children Fund;

PROSCAP $=$ Promotion of Soil Conservation and Rural Production; WB $=$ World Bank; nk = not known.

revealed similar results, with most levels found to be below $2 \mathrm{mg} / \ell$. The highest recorded levels were between 46 to $58 \mathrm{mg} / \ell$.

Most chemicals arising in drinking-water are of health concern only after extended exposure of years, rather than months. The principal exception is nitrate. The presence of excessive nitrate and nitrite in water has been associated with methaemoglobinaemia (blue baby syndrome), especially in bottled fed infants. Bottle-fed infants are considered to be at greater risk because the intake of water in relation to body weight is high and, in infants, the development of repair enzymes is limited (WHO, 2008). In clinical epidemiological studies of methaemoglobinaemia and subclinical increases in methaemoglobin associated with drinking-water nitrate, $97 \%$ of cases occurred at concentrations in excess of $44.3 \mathrm{mg} / \ell$, with clinical symptoms associated with the higher concentrations (WHO, 2008).

The risk of methaemoglobinaemia in infants significantly increases with simultaneous exposure to nitrates and microbial contaminants in water and is rarely associated with nitrate in the absence of faecal contamination of drinking-water (WHO, 2008). This is of concern as faecal coliform and/or enterococci bacteria were detected in just over half of borehole water supplies tested at the pump, including Mfela School (2-120 E. coli $\mathrm{cfu} / 100 \mathrm{~m} \ell ; 12-22$ enterococci cfu/100 m $\ell ; n=3$ ) (Grimason et al., 2013). Coliform or enterococci bacteria were not detected in borehole water samples from Khumbulani, Wiliyamu I and Migano.

As a short-term measure, the WHO recommend that water should not be used for bottle-fed infants when nitrate levels are above $100 \mathrm{mg} / \ell$; however, it may be used if medical authorities are increasingly vigilant when the nitrate concentration is between 50 and $100 \mathrm{mg} / \ell$, provided that the water is known and is confirmed to be microbially safe (WHO, 2008). Contamination of aquifers in this study may have arisen from the application of organic and inorganic fertilisers used by the sugar plantation industry, natural fertilisers (animal waste, plant material) used by local farmers and/or from leaching of wastewater from inappropriately sited pit latrines. To reduce the potential for nitrate contamination of aquifers there is need for large and small-scale farmers to adopt good agricultural practice and water committees to implement water safety plans at village level. There is also the need to emphasise to young mothers the importance of boiling borehole water prior to reconstituting baby foods for bottle-fed infants. 
TABLE 3

Mean concentration ( + SD) of chemical constituents in borehole water samples collected from the east bank of the Shire River

\begin{tabular}{|c|c|c|c|c|c|c|c|c|c|c|c|c|c|c|}
\hline BHNo. & Villag & $\begin{array}{c}\mathrm{Ca} \\
(\mathrm{mg} / \mathrm{l})\end{array}$ & $\underset{(\mathrm{mg} / \mathrm{l})}{\mathrm{Cu}}$ & $\begin{array}{c}\mathrm{Cr} \\
(\mu \mathrm{g} / \ell)\end{array}$ & $\begin{array}{c}\mathrm{Fe} \\
(\mathrm{mg} / \mathrm{\ell})\end{array}$ & $\begin{array}{c}\mathrm{Mn} \\
(\mathrm{mg} / \mathrm{\ell})\end{array}$ & $\underset{(\mathrm{mg} / \mathrm{l})}{\mathrm{Mg}}$ & $\underset{(\mathrm{mg} / \mathrm{l})}{\mathrm{Zn}}$ & $\begin{array}{c}\mathrm{K} \\
(\mathrm{mg} / \mathrm{l})\end{array}$ & $\begin{array}{c}\mathrm{Na} \\
(\mathrm{mg} / \mathrm{l})\end{array}$ & $\begin{array}{c}\mathrm{SO}_{4} \\
(\mathrm{mg} / \mathrm{\ell})\end{array}$ & $\begin{array}{c}\mathrm{NO}_{3} \\
(\mathrm{mg} / \mathrm{l})\end{array}$ & $\begin{array}{c}\mathrm{F} \\
(\mathrm{mg} / \mathrm{\ell})\end{array}$ & $\begin{array}{c}\mathrm{Cl} \\
(\mathrm{mg} / \mathrm{\ell})\end{array}$ \\
\hline 1 & Mkhwicho & $\begin{array}{c}35.9 \pm \\
6.4\end{array}$ & $\begin{array}{c}0.1 \pm \\
0.1\end{array}$ & $\begin{array}{c}0.7 \pm \\
0.6\end{array}$ & 0.2 & 0.0 & 5.6 & & $\begin{array}{c}1.4 \pm \\
0.1\end{array}$ & $\begin{array}{c}50.5 \pm \\
2.4\end{array}$ & $\begin{array}{c}37.8 \pm \\
1.1\end{array}$ & $\begin{array}{c}3.3 \pm \\
0.5\end{array}$ & 0.1 & $\begin{array}{c}21 \pm \\
1.3\end{array}$ \\
\hline 2 & Matumula & $\begin{array}{c}57.6 \pm \\
4.4\end{array}$ & $\begin{array}{c}0.3 \pm \\
0.3\end{array}$ & $\begin{array}{c}1.0 \pm \\
1.0\end{array}$ & $\begin{array}{c}0.3 \pm \\
0.3\end{array}$ & 0 . & $\begin{array}{c}19.8 \pm \\
1.9\end{array}$ & $\begin{array}{c}0.3 \pm \\
0.4\end{array}$ & $\begin{array}{c}6.3 \pm \\
0.7\end{array}$ & $\begin{array}{c}17.3 \pm \\
0.8\end{array}$ & $\begin{array}{c}18.1 \pm \\
1.5\end{array}$ & $\begin{array}{c}29.1 \pm \\
2.6\end{array}$ & $\begin{array}{c}0.1 \pm \\
0.2\end{array}$ & $\begin{array}{c}8.1 \pm \\
1.5\end{array}$ \\
\hline 3 & Mpokonyola & $\begin{array}{c}20.3 \pm \\
3.5\end{array}$ & $\begin{array}{c}0.1 \pm \\
0.1\end{array}$ & $\begin{array}{c}0.7 \pm \\
0.6\end{array}$ & $\begin{array}{c}0.4 \pm \\
0.3\end{array}$ & $\begin{array}{c}0.2 \pm \\
0.0\end{array}$ & $\begin{array}{c}23.1 \pm \\
3.1\end{array}$ & $\begin{array}{c}0.1 \pm \\
0.1\end{array}$ & $\begin{array}{c}2.2 \pm \\
0.3\end{array}$ & $\begin{array}{c}15.0 \pm \\
3.2\end{array}$ & $\begin{array}{c}6.5 \pm \\
1.3\end{array}$ & $\begin{array}{c}9.6 \pm \\
3.8\end{array}$ & $\begin{array}{c}0.3 \pm \\
0.6\end{array}$ & $\begin{array}{c}31.9 \pm \\
17.2\end{array}$ \\
\hline 4 & Mfel & $\begin{array}{c}37.5 \pm \\
11.9\end{array}$ & $\begin{array}{c}0.1 \pm \\
0.1\end{array}$ & $\begin{array}{c}0.7 \pm \\
0.6\end{array}$ & $\begin{array}{c}0.4 \pm \\
0.3\end{array}$ & $\begin{array}{c}0.3 \pm \\
0.2\end{array}$ & $\begin{array}{c}24.9 \pm \\
11.8\end{array}$ & 0 . & $\begin{array}{c}2.0 \pm \\
0.7\end{array}$ & $\begin{array}{l}15.6 \\
\pm 3\end{array}$ & $\begin{array}{l}15.9 \\
+5\end{array}$ & $\begin{array}{c}42.3 \pm \\
0.7\end{array}$ & $\begin{array}{c}0.4 \pm \\
0.1\end{array}$ & $\begin{array}{c}33.7 \pm \\
20.2\end{array}$ \\
\hline 5 & Kapt & $\begin{array}{c}26.0 \pm \\
2.9\end{array}$ & $\begin{array}{c}0.3 \pm \\
0.2\end{array}$ & $\begin{array}{c}1.3 \pm \\
1.2\end{array}$ & $\begin{array}{c}0.6 \pm \\
0.4\end{array}$ & $\begin{array}{c}0.4 \pm \\
0.0\end{array}$ & $\begin{array}{c}13.7 \pm \\
0.2\end{array}$ & $\begin{array}{c}0.3 \pm \\
0.2\end{array}$ & $\begin{array}{c}1.5 \pm \\
1.3\end{array}$ & $\begin{array}{c}16.0 \pm \\
1.6\end{array}$ & $\begin{array}{c}5.9 \pm \\
0.5\end{array}$ & $\begin{array}{c}0.1 \pm \\
0.1\end{array}$ & $\begin{array}{c}4.8 \pm \\
0.5\end{array}$ & $\begin{array}{c}8.9 \pm \\
2.3\end{array}$ \\
\hline 6 & Khumbulani & $\begin{array}{c}88.6 \pm \\
75.8\end{array}$ & $\begin{array}{c}1.0 \pm \\
0.8\end{array}$ & $\begin{array}{c}0.7 \pm \\
0.6\end{array}$ & $\begin{array}{c}0.6 \pm \\
0.5\end{array}$ & 0. & $\begin{array}{c}35.8 \pm \\
6.2\end{array}$ & $\begin{array}{c}0.8 \pm \\
0.7\end{array}$ & $\begin{array}{c}2.4 \pm \\
0.2\end{array}$ & $\begin{array}{c}63.5 \pm \\
12.1\end{array}$ & $\begin{array}{c}7.9 \pm \\
0.3\end{array}$ & $\begin{array}{c}24.7 \pm \\
0.5\end{array}$ & $\begin{array}{c}0.4 \pm \\
0.3\end{array}$ & $\begin{array}{c}12.7 \pm \\
1.7\end{array}$ \\
\hline 7 & C & $\begin{array}{c}55.9 \pm \\
7.0\end{array}$ & $\begin{array}{c}0.1 \pm \\
0.0\end{array}$ & $\begin{array}{c}0.7 \pm \\
0.6\end{array}$ & $\begin{array}{c}0.4 \pm \\
0.3\end{array}$ & 0.0 & $\begin{array}{c}32.5 \pm \\
2.5\end{array}$ & $\begin{array}{c}0.1 \pm \\
0.1\end{array}$ & $\begin{array}{c}3.3 \pm \\
0.1\end{array}$ & $\begin{array}{c}85.4 \pm \\
24.5\end{array}$ & $\begin{array}{l}17.2 \\
\pm 1\end{array}$ & $\begin{array}{c}27.8 \pm \\
4.3\end{array}$ & $\begin{array}{c}0.5 \pm \\
0.6\end{array}$ & $\begin{array}{c}60.2 \pm \\
38.6\end{array}$ \\
\hline 8 & lani & $\begin{array}{c}43.4 \pm \\
7.8\end{array}$ & $\begin{array}{c}0.1 \pm \\
0.0\end{array}$ & $\begin{array}{c}0.7 \pm \\
0.6\end{array}$ & $\begin{array}{c}0.4 \pm \\
0.0\end{array}$ & $\begin{array}{c}0.1 \pm \\
0.1\end{array}$ & $\begin{array}{c}30.8 \pm \\
3.6\end{array}$ & $\begin{array}{c}0.3 \pm \\
0.1\end{array}$ & $\begin{array}{c}2.5 \pm \\
0.2\end{array}$ & $\begin{array}{c}50.6 \pm \\
0.7\end{array}$ & $\begin{array}{c}14.8 \pm \\
1.8\end{array}$ & $\begin{array}{c}47.3 \pm \\
6.3\end{array}$ & $\begin{array}{c}1.9 \pm \\
0.6\end{array}$ & $\begin{array}{c}16.7 \pm \\
1.6\end{array}$ \\
\hline 9 & Zimp & $\begin{array}{c}83.7 \pm \\
65.5\end{array}$ & $\begin{array}{c}0.0 \pm \\
0.1\end{array}$ & $\begin{array}{c}1.2 \pm \\
1.0\end{array}$ & $\begin{array}{c}0.2 \pm \\
0.1\end{array}$ & 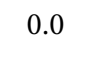 & $\begin{array}{c}25.6 \pm \\
1.8\end{array}$ & $\begin{array}{c}0.0 \pm \\
0.1\end{array}$ & $\begin{array}{c}1.0 \pm \\
0.6\end{array}$ & $\begin{array}{c}43.7 \pm \\
1.1\end{array}$ & $\begin{array}{c}45.9 \pm \\
10.4\end{array}$ & 0.0 & $\begin{array}{c}0.6 \pm \\
0.2\end{array}$ & $\begin{array}{c}71.2 \pm \\
6.9\end{array}$ \\
\hline 10 & $S$ & $\begin{array}{c}40.0 \pm \\
8.1\end{array}$ & $\begin{array}{c}0.0 \pm \\
0.1\end{array}$ & $\begin{array}{c}0.7 \pm \\
0.6\end{array}$ & $\begin{array}{c}0.4 \pm \\
0.3\end{array}$ & 0.0 & $\begin{array}{c}40.9 \pm \\
14.4\end{array}$ & $\begin{array}{c}0.1 \pm \\
0.1\end{array}$ & $\begin{array}{c}1.4 \pm \\
0.2\end{array}$ & $\begin{array}{c}24.1 \pm \\
6.8\end{array}$ & $\begin{array}{c}14.6 \pm \\
7.6\end{array}$ & $\begin{array}{c}19.9 \pm \\
11.8\end{array}$ & $\begin{array}{c}0.4 \pm \\
0.2\end{array}$ & $\begin{array}{c}16.3 \pm \\
18.7\end{array}$ \\
\hline 11 & $\begin{array}{l}\text { A } \\
\mathrm{N}\end{array}$ & $\begin{array}{c}111.8 \pm \\
42.6\end{array}$ & $\begin{array}{c}0.1 \pm \\
0.0\end{array}$ & $\begin{array}{c}0.7 \pm \\
0.6\end{array}$ & $\begin{array}{c}0.6 \pm \\
0.1\end{array}$ & 0. & $\begin{array}{c}32.7 \pm \\
2.4\end{array}$ & $\begin{array}{c}0.1 \pm \\
0.1\end{array}$ & $\begin{array}{c}0.5 \pm \\
0.1\end{array}$ & $\begin{array}{c}52.5 \pm \\
18\end{array}$ & $\begin{array}{c}12.1 \pm \\
0.2\end{array}$ & $\begin{array}{c}8.5 \pm \\
0.3\end{array}$ & $\begin{array}{c}0.4 \pm \\
0.5\end{array}$ & $\begin{array}{c}29.5 \pm \\
11.1\end{array}$ \\
\hline 12 & Mpar & $\begin{array}{c}40.0 \pm \\
1.4\end{array}$ & 0.0 & $\begin{array}{c}1.0 \pm \\
1.0\end{array}$ & $\begin{array}{c}1.1 \pm \\
0.9\end{array}$ & $\begin{array}{c}0.2 \pm \\
0.1\end{array}$ & $\begin{array}{c}48.7 \pm \\
3.1\end{array}$ & 0 . & $\begin{array}{c}2.4 \pm \\
0.2\end{array}$ & $\begin{array}{c}97.2 \pm \\
1.5\end{array}$ & $\begin{array}{c}6.6 \pm \\
0.7\end{array}$ & $\begin{array}{c}3.7 \pm \\
1.6\end{array}$ & $\begin{array}{c}0.9 \pm \\
0.2\end{array}$ & $\begin{array}{c}22.1 \pm \\
6.6\end{array}$ \\
\hline 13 & Bello & $\begin{array}{c}31.8 \pm \\
0.5\end{array}$ & 0.0 & $\begin{array}{c}1.0 \pm \\
1.0\end{array}$ & $\begin{array}{c}0.5 \pm \\
0.4\end{array}$ & $\begin{array}{c}0.5 \pm \\
0.1\end{array}$ & $\begin{array}{c}15.9 \pm \\
0.7\end{array}$ & $\begin{array}{c}0.0 \pm \\
0.1\end{array}$ & $\begin{array}{c}1.8 \pm \\
0.2\end{array}$ & $\begin{array}{c}30.4 \pm \\
0.7\end{array}$ & $\begin{array}{c}9.4 \pm \\
0.6\end{array}$ & 0.0 & $\begin{array}{c}0.2 \pm \\
0.1\end{array}$ & $\begin{array}{c}46.4 \pm \\
29\end{array}$ \\
\hline 14 & Chadula I & $\begin{array}{c}25.4 \pm \\
2.0\end{array}$ & $\begin{array}{c}0.2 \\
\pm 0.2\end{array}$ & $\begin{array}{c}1.0 \pm \\
1.0\end{array}$ & $\begin{array}{c}1.6 \pm \\
0.4\end{array}$ & $\begin{array}{c}0.2 \pm \\
0.1\end{array}$ & $\begin{array}{c}12.8 \pm \\
1.2\end{array}$ & $\begin{array}{c}0.1 \pm \\
0.1\end{array}$ & $\begin{array}{c}2.0 \pm \\
0.2\end{array}$ & $\begin{array}{c}18.8 \pm \\
1.5\end{array}$ & $\begin{array}{c}4.5 \pm \\
1\end{array}$ & $\begin{array}{c}1.1 \pm \\
1.8\end{array}$ & $\begin{array}{c}0.2 \pm \\
0.1\end{array}$ & $\begin{array}{c}8.4 \pm \\
1.9\end{array}$ \\
\hline 15 & Chadula II & $\begin{array}{c}28.5 \pm \\
5.1\end{array}$ & $\begin{array}{c}0.0 \\
\pm 0.1\end{array}$ & $\begin{array}{c}0.7 \pm \\
0.6\end{array}$ & $\begin{array}{c}1.0 \pm \\
0.7\end{array}$ & $\begin{array}{c}0.5 \pm \\
0.0\end{array}$ & $\begin{array}{c}13.5 \pm \\
2.6\end{array}$ & 0.0 & $\begin{array}{c}1.8 \pm \\
02\end{array}$ & $\begin{array}{c}29.2 \pm \\
2.1\end{array}$ & $\begin{array}{c}10.3 \pm \\
0.6\end{array}$ & 0.0 & $\begin{array}{c}0.6 \pm \\
0.2\end{array}$ & $\begin{array}{c}15.8 \pm \\
2.5\end{array}$ \\
\hline 16 & Mtuwawa & $\begin{array}{c}27.8 \pm \\
0.5\end{array}$ & $\begin{array}{c}0.3 \\
\pm 0.6\end{array}$ & $\begin{array}{c}0.7 \pm \\
0.6\end{array}$ & $\begin{array}{c}1.3 \pm \\
0.3\end{array}$ & $\begin{array}{c}0.6 \pm \\
0.1\end{array}$ & $\begin{array}{c}12.8 \pm \\
0.4\end{array}$ & 0.0 & $\begin{array}{c}2.1 \pm \\
0.1\end{array}$ & $\begin{array}{c}31.6 \pm \\
0.3\end{array}$ & $\begin{array}{c}11.2 \pm \\
0.8\end{array}$ & $\begin{array}{c}20.4 \pm \\
1.9\end{array}$ & $\begin{array}{c}0.3 \pm \\
0.2\end{array}$ & $\begin{array}{c}39.2 \pm \\
22.7\end{array}$ \\
\hline
\end{tabular}

All borehole samples contained sulphate concentrations well below the MBS MPL. Three boreholes located on the west bank were found to contain considerably higher concentrations of sulphates, i.e. Dyelatu School, Migano and Kanthema. The low concentrations of sulphates found on the east bank are similar to those reported by Sajidu et al. (2007), who assessed borehole water quality during the rainy season in Machinga (range $<10-110 \mathrm{mg} / \ell$ ), and Bath (1980), who investigated the quality of groundwaters in north (South Rukuru $<15 \mathrm{mg} / \ell$ ) and central (Nkhotakota $<2400 \mathrm{mg} / \ell$ ) regions of Malawi.

The presence of sulphate in drinking-water can cause a noticeable taste, and very high levels might cause a laxative effect in unaccustomed consumers (WHO, 2008). As such, the WHO recommends that health authorities be notified of sources of drinking-water that contain sulphate concentrations $>500 \mathrm{mg} / \ell$. Taste impairment varies with the nature of the associated cation; taste thresholds have been found to range from $250 \mathrm{mg} / \ell$ for sodium sulphate to $1000 \mathrm{mg} / \ell$ for calcium sulphate. It is generally considered that taste impairment is minimal at levels below $250 \mathrm{mg} / \ell$. However, zinc sulphate imparts an undesirable astringent taste at a threshold concentration of about $4 \mathrm{mg} / \ell$ (WHO, 2008), which may be associated with consumer complaints related to 'bitterness', as expressed by some water committee members. No WHO health-based GV has been set for sulphate.

Fluoride concentrations in borehole samples on the east bank of the river ranged from 0 to $5 \mathrm{mg} / \ell$ (median: $0.5 \mathrm{mg} / \mathrm{\ell}$ ) and on the west bank from 1 to $3.4 \mathrm{mg} / \ell$. However, the majority $(81 \%)$ of samples on the east bank (13 villages) were $<1.0 \mathrm{mg} / \ell$. Fluoride levels $>1.0 \mathrm{mg} / \ell$ were recorded at 2 boreholes on the east bank, i.e., Mkhwicho and Khumbulani. The majority (60\%) of borehole samples on the west bank (7 villages) were within the range of 1.5 to $3 \mathrm{mg} / \ell$. Lower concentrations were detected at Wiliyamu I and II and higher concentrations at Migno, Kabudula and Lakiuji, respectively. All samples were within the MBS MPL.

Fluoride in water is considered to be an essential element for the development and protection of teeth and bones. The 
TABLE 4

Mean concentration (+ SD) of chemical constituents in borehole water samples collected from the west bank of the Shire River

\begin{tabular}{|c|c|c|c|c|c|c|c|c|c|c|c|c|c|c|}
\hline BHNo. & Village/Area & $\begin{array}{c}\mathrm{Ca} \\
(\mathrm{mg} / \mathrm{l})\end{array}$ & $\underset{(\mathrm{mg} / \ell)}{\mathrm{Cu}}$ & $\begin{array}{c}\mathrm{Cr} \\
(\mu \mathrm{g} / \ell)\end{array}$ & $\begin{array}{c}\mathrm{Fe} \\
(\mathrm{mg} / \mathrm{l})\end{array}$ & $\begin{array}{c}\mathrm{Mn} \\
(\mathrm{mg} / \mathrm{l})\end{array}$ & $\underset{(\mathrm{mg} / \mathrm{l})}{\mathrm{Mg}}$ & $\begin{array}{c}\mathrm{Zn} \\
(\mathrm{mg} / \ell)\end{array}$ & $\begin{array}{c}\mathrm{K} \\
(\mathrm{mg} / \ell)\end{array}$ & $\begin{array}{c}\mathrm{Na} \\
(\mathrm{mg} / \mathrm{l})\end{array}$ & $\begin{array}{c}\mathrm{SO}_{4} \\
(\mathrm{mg} / \mathrm{l})\end{array}$ & $\begin{array}{c}\mathrm{NO}_{3} \\
(\mathrm{mg} / \mathrm{l})\end{array}$ & $\underset{(\mathrm{mg} / \ell)}{\mathrm{F}}$ & $\begin{array}{c}\mathrm{Cl} \\
(\mathrm{mg} / \mathrm{l})\end{array}$ \\
\hline 17 & Wiliyamu I & $\begin{array}{c}83.4 \pm \\
24.7\end{array}$ & $\begin{array}{c}0.1 \pm \\
0.1\end{array}$ & $\begin{array}{c}1.3 \pm \\
0.6\end{array}$ & $\begin{array}{c}0.8 \pm \\
0.6\end{array}$ & 0 & $\begin{array}{c}36 \pm \\
0.6\end{array}$ & $\begin{array}{c}0.1 \pm \\
0.1\end{array}$ & $\begin{array}{c}4.4 \pm \\
0.9\end{array}$ & $\begin{array}{l}45.3 \\
\pm 3\end{array}$ & $\begin{array}{c}16.3 \\
\pm 6\end{array}$ & $\begin{array}{l}47 \pm \\
15.3\end{array}$ & $\begin{array}{l}1 \pm \\
0.1\end{array}$ & $38 \pm 32$ \\
\hline 18 & Wiliyamu II & $\begin{array}{c}34 \pm \\
3.1\end{array}$ & 0 & $\begin{array}{c}0.8 \pm \\
0.7\end{array}$ & $\begin{array}{c}0.8 \pm \\
0.1\end{array}$ & 0 & $\begin{array}{c}17.8 \pm \\
3.3\end{array}$ & 0 & $\begin{array}{c}5.2 \pm \\
0.7\end{array}$ & $\begin{array}{c}329.7 \pm \\
25.6\end{array}$ & \begin{tabular}{|c}
$59.6 \pm$ \\
7.9
\end{tabular} & $\begin{array}{c}5.5 \pm \\
0.4\end{array}$ & $\begin{array}{c}1.4 \pm \\
0.1\end{array}$ & $\begin{array}{c}68.9 \pm \\
58.5\end{array}$ \\
\hline 19 & Nedi & $\begin{array}{c}120.5 \pm \\
37.9\end{array}$ & 0 & $\begin{array}{c}0.7 \pm \\
0.6\end{array}$ & $\begin{array}{c}0.4 \pm \\
0.3\end{array}$ & 0 & $\begin{array}{l}54 \pm \\
14.6\end{array}$ & 0 & $\begin{array}{c}4.9 \pm \\
0.4\end{array}$ & $\begin{array}{c}401.9 \pm \\
40.7\end{array}$ & $\begin{array}{c}75.4 \pm \\
2.6\end{array}$ & $\begin{array}{c}37 \pm \\
0.6\end{array}$ & $\begin{array}{c}2.2 \pm \\
0.3\end{array}$ & $\begin{array}{l}386 \pm \\
173.6\end{array}$ \\
\hline 20 & Kanthema & $\begin{array}{c}63.0 \pm \\
7.7\end{array}$ & 0 & $\begin{array}{c}0.6 \pm \\
0.6\end{array}$ & $\begin{array}{c}0.4 \pm \\
0.2\end{array}$ & 0 & $\begin{array}{c}42.9 \pm \\
3.8\end{array}$ & 0 & $\begin{array}{c}3.5 \pm \\
0.1\end{array}$ & $\begin{array}{c}366 \pm \\
24.1\end{array}$ & $\begin{array}{c}781.7 \\
\pm 2\end{array}$ & $\begin{array}{c}7.3 \pm \\
0.6\end{array}$ & $\begin{array}{c}2.1 \pm \\
0.2\end{array}$ & $\begin{array}{c}145.9 \pm \\
10.2\end{array}$ \\
\hline 21 & Kabudula I & $\begin{array}{c}22.4 \\
\pm 8\end{array}$ & 0 & $\begin{array}{c}0.7 \pm \\
0.6\end{array}$ & $\begin{array}{c}0.5 \pm \\
0.3\end{array}$ & 0 & $\begin{array}{c}21.6 \\
\pm 9\end{array}$ & 0 & $\begin{array}{c}4.6 \pm \\
0.2\end{array}$ & $\begin{array}{c}474.8 \pm \\
60.7\end{array}$ & $\begin{array}{c}84.4 \pm \\
34.7\end{array}$ & $\begin{array}{c}29.9 \pm \\
25.9\end{array}$ & $\begin{array}{c}1.6 \pm \\
0.2\end{array}$ & $\begin{array}{c}142.3 \pm \\
54.5\end{array}$ \\
\hline 22 & Kabudula II & $\begin{array}{c}31.5 \pm \\
12\end{array}$ & 0 & $\begin{array}{c}0.7 \pm \\
0.6\end{array}$ & $\begin{array}{c}0.6 \pm \\
0.4\end{array}$ & 0 & $\begin{array}{c}16.8 \pm \\
13\end{array}$ & 0 & $\begin{array}{c}4.1 \pm \\
0.8\end{array}$ & $\begin{array}{c}519.1 \pm \\
43.9\end{array}$ & $\begin{array}{c}62.8 \pm \\
31.9\end{array}$ & 0 & $\begin{array}{c}3.2 \pm \\
0.3\end{array}$ & $\begin{array}{c}103.8 \pm \\
3.1\end{array}$ \\
\hline 23 & Lawyi 2 & $\begin{array}{c}31.6 \pm \\
4.9\end{array}$ & 0 & $\begin{array}{c}0.7 \pm \\
0.6\end{array}$ & $\begin{array}{c}1.0 \pm \\
0.8\end{array}$ & 0 & $\begin{array}{c}18.1 \pm \\
2.7\end{array}$ & 0 & $\begin{array}{l}5 \pm \\
0.3\end{array}$ & $\begin{array}{c}574.1 \pm \\
43.8\end{array}$ & $\begin{array}{c}87.6 \pm \\
5.6\end{array}$ & 0 & $\begin{array}{l}2 \pm \\
0.2\end{array}$ & $\begin{array}{c}90.9 \pm \\
4.3\end{array}$ \\
\hline 24 & Dyelatu School & $\begin{array}{c}96.5 \pm \\
14.3\end{array}$ & $\begin{array}{c}0.1 \pm \\
0.1\end{array}$ & $\begin{array}{c}0.7 \pm \\
0.6\end{array}$ & $\begin{array}{c}1.5 \pm \\
1.1\end{array}$ & $\begin{array}{c}0.1 \pm \\
0.1\end{array}$ & $\begin{array}{l}23 \pm \\
15.5\end{array}$ & 0 & $\begin{array}{c}16.3 \pm \\
6.8\end{array}$ & $\begin{array}{c}531.7 \pm \\
1.6\end{array}$ & $\begin{array}{c}240.5 \pm \\
18.7\end{array}$ & 0 & $\begin{array}{c}2.8 \pm \\
0.7\end{array}$ & $\begin{array}{c}176.7 \pm \\
3.2\end{array}$ \\
\hline 25 & Lakiuji & $\begin{array}{c}99.7 \pm \\
41.6\end{array}$ & $\begin{array}{c}0.1 \pm \\
0.0\end{array}$ & $\begin{array}{c}1.3 \pm \\
1.2\end{array}$ & $\begin{array}{c}1.6 \pm \\
0.2\end{array}$ & $\begin{array}{c}0.1 \pm \\
0.1\end{array}$ & $\begin{array}{c}32.7 \pm \\
21\end{array}$ & $\begin{array}{c}0.1 \pm \\
0.0\end{array}$ & $\begin{array}{c}24.3 \pm \\
13.8\end{array}$ & $\begin{array}{c}613.6 \pm \\
387\end{array}$ & $\begin{array}{c}181.3 \pm \\
222\end{array}$ & $\begin{array}{c}15.4 \pm \\
13.3\end{array}$ & $\begin{array}{c}3.9 \pm \\
0.5\end{array}$ & $\begin{array}{c}156.6 \pm \\
68.2\end{array}$ \\
\hline 26 & Migano & $\begin{array}{c}104.9 \pm \\
11.6\end{array}$ & $\begin{array}{c}0.1 \pm \\
0.0\end{array}$ & $\begin{array}{c}1.3 \pm \\
1.2\end{array}$ & $\begin{array}{c}3.9 \pm \\
3.2\end{array}$ & $\begin{array}{c}0.2 \pm \\
0.1\end{array}$ & $\begin{array}{c}54.3 \pm \\
5.2\end{array}$ & $\begin{array}{c}0.1 \pm \\
0.1\end{array}$ & $\begin{array}{c}23.4 \pm \\
3.2\end{array}$ & $\begin{array}{l}1177.6 \\
\pm 29.1 \\
\end{array}$ & $\begin{array}{c}294.4 \pm \\
11\end{array}$ & $\begin{array}{c}177.6 \pm \\
23\end{array}$ & $\begin{array}{l}3 \pm \\
0.3\end{array}$ & $\begin{array}{c}330.3 \pm \\
44.1\end{array}$ \\
\hline 27 & Sisev & $\begin{array}{r}25.6 \\
\pm 4 \\
\end{array}$ & $\begin{array}{c}0.4 \pm \\
0.7\end{array}$ & $\begin{array}{c}0.7 \pm \\
0.6 \\
\end{array}$ & $\begin{array}{c}0.5 \pm \\
0.4 \\
\end{array}$ & 0 & $\begin{array}{c}27.6 \pm \\
2.4\end{array}$ & 0 & $\begin{array}{c}19.1 \pm \\
1.3\end{array}$ & $\begin{array}{c}154.6 \pm \\
16.8\end{array}$ & $\begin{array}{c}24.9 \pm \\
0.6\end{array}$ & $\begin{array}{c}28.7 \pm \\
24.9\end{array}$ & $\begin{array}{c}2.8 \pm \\
0.3\end{array}$ & $\begin{array}{c}34.5 \pm \\
4.7\end{array}$ \\
\hline 28 & Mtondeza & $\begin{array}{c}32.8 \pm \\
1.6\end{array}$ & 0 & $\begin{array}{c}0.7 \pm \\
0.6\end{array}$ & $\begin{array}{c}0.9 \pm \\
0.1\end{array}$ & $\begin{array}{c}0.4 \pm \\
0.4\end{array}$ & $\begin{array}{c}13.8 \pm \\
5.4\end{array}$ & 0 & $\begin{array}{c}9.8 \pm \\
0.5\end{array}$ & $\begin{array}{c}697.2 \pm \\
322\end{array}$ & $\begin{array}{c}154.3 \pm \\
160\end{array}$ & $\begin{array}{l}3 \pm \\
5.2\end{array}$ & $\begin{array}{c}2.9 \pm \\
0.5\end{array}$ & $\begin{array}{c}426 \pm \\
89\end{array}$ \\
\hline
\end{tabular}

current WHO health-based GV is considered to be a threshold where the benefit of resistance to tooth decay is weighed against the risk of developing dental fluorisis. In comparison, the USEPA has set two standards for fluoride: a non-mandatory Secondary Drinking Water Standard (SDWS) of $2 \mathrm{mg} / \ell$ to prevent dental fluorosis and a primary drinking water standard (PDWS) of $4 \mathrm{mg} / \ell$ to protect against skeletal fluorosis (EPA, 2001). The basis for the MBS MPL being 4 times greater than the WHO GV and 3 and $1 \frac{1}{2}$ times greater than the USEPA SDWS and PDWS's, respectively, is unclear and clarification could not be provided by the MBS when consulted.

In this study, levels $\geq 2 \mathrm{mg} / \ell$ were detected at 2 boreholes on the east bank and 8 boreholes on the west bank. In addition, levels $\geq 4 \mathrm{mg} / \ell$ were detected in one borehole supply on each side of the Shire River (Lakiuji on the west bank and Kapufeni on the east bank). Dental fluorosis is characterised by discoloured, blackened, mottled or chalky-white teeth. This was visibly evident amongst members of the water committees and collectors in villages located on the west bank and less so amongst members of the water committees and collectors from villages on the east bank (pers. observation).

In 2008, Sajidu et al. undertook a detailed study of groundwater fluoride levels in 49 villages from 5 districts in Southern Malawi. Two out of five samples from boreholes located in Chikhwawa exceeded (1.91-1.93 mg/ $/$ ) the WHO GV, but were within the higher levels laid down in the US and MBS standards. In Nsanje, a neighbouring District to Chikhwawa, only one of six borehole samples complied with the WHO GV, three with the US SDWS, four with the US PDWS and five with the MBS MPL (maximum $7.25 \mathrm{mg} / \ell$ ). This area is renowned within the country for the sight of dental fluorosis amongst locals.

All borehole samples analysed for chloride had levels below the MBS MPL, although marked differences were observed between the relatively low concentrations detected on the east (range 5.1-91.6 mg/ $/$ ) compared with the elevated concentrations detected on the west bank of the river (range 1.5-493.8 $\mathrm{mg} / \ell$ ). Three villages on the west bank of the river had borehole supplies with chloride concentrations in excess of $250 \mathrm{mg} / \ell$ (Nedi, Migano and Mtondeza). Much higher concentrations of chloride in groundwater from the Lower Shire have been reported in earlier studies. Bath (1980) reported finding levels between $2100 \mathrm{mg} / \ell$ and $4000 \mathrm{mg} / \ell$ in borehole water, rendering the water unpalatable, with the result that the water source was abandoned and alternative unprotected sources utilised. In contrast, lower chloride concentrations were found by Sajidu et al. (2007) in borehole water in Machinga during the rainy season $(0.03-0.57 \mathrm{mg} / \ell)$.

A health-based guideline for chloride in drinking water has not been established by the WHO as it is deemed to be a naturally occurring chemical which is usually found at concentrations which are not toxic to health (WHO, 2008). However, it is acknowledged that concentrations above $250 \mathrm{mg} / \ell \mathrm{can}$ give rise to a detectable 'salty' taste in water. In this study, 3 villages had borehole water quality which exceeded $250 \mathrm{mg} / \mathrm{\ell}$ (Nedi, Migano and Mtondeza). Water committee members from all three villages stated that the salty taste of the water is a 
common complaint of villagers, but acknowledged that overall the quality of water was better than that from other sources, such as the river and unprotected shallow wells. One water committee member from Mtondeza stated that although she collected borehole water for 'her children, grandchildren and herself for drinking purposes, she also collected river water for her husband as he did not like the taste of the borehole water'. Problems associated with high salinity in groundwater in the Lower Shire are well recognised (Bath, 1980).

Sodium concentrations varied markedly in water samples from the east and west bank of the river. On the east bank every borehole had concentrations below $200 \mathrm{mg} / \ell$, well within the MBS MPL. On the west bank considerably higher concentrations were detected (median $470.5 \mathrm{mg} / \ell$ ). Six borehole samples were below the MBS MPL and 6 were found to exceed it at significantly higher levels at Kabudula II, Lawyi 2, Dyelatu School, Lakiuji, Mtondeza and Migano.

Water committee members from two of the six villages complained about the 'saltiness' of the water (Migano and Mtondeza), as did committee members from Nedi village. The 'salty' taste associated with these borehole samples may be due to the salinity of the aquifer as each of the water samples also had significantly high chloride levels. It may be that water committee members from the other three villages had developed a tolerance for the sodium-chloride rich waters (WHO, 2008).

Calcium concentrations in borehole water supplies from both banks of the Shire River complied with the MBS MPL. The maximum concentration detected on the east bank was detected at Admarc Market and on the west bank at Nedi. Nedi also had the second-highest chloride level recorded in the study and was the source of many consumer complaints due to the 'saltiness' of the water. The second-highest concentration of calcium was recorded at Migano, which also had high chloride levels and was the source of similar consumer complaints. Given the results observed in this study, the salty taste imparted to the water may be due to high concentrations of sodium and calcium chloride. No health-based equivalent guideline value for calcium has been established by the WHO for comparison.

Saltiness can lead to borehole supplies being abandoned as a source of drinking-water and used for domestic chores only, such as washing clothes. As a result, village inhabitants become despondent at being provided with a borehole that does not fulfil their needs and expectations, and borehole construction is deemed to have been a waste of energy, time and valuable human and financial resources. This usually results in the inhabitants resorting to the use of unprotected sources of water supply, such as river water, for their drinking-water (pers. observation).

In general, dissolved iron levels detected ranged from $0.3 \mathrm{mg} / \ell$ to $2 \mathrm{mg} / \ell$, which is well below the MBS MPL. The only borehole supply to breach the MBS MPL standard was Magano $(3.9 \mathrm{mg} / \ell)$. These concentrations, although slightly higher than those normally found in groundwater (WHO, 2003b), are in accordance with earlier studies undertaken on groundwater sources in Malawi (Bath, 1980; UN, 1989). Earlier studies conducted in Malawi present data on total (unfiltered) iron levels in groundwater samples. For example, total iron levels of up to $84 \mathrm{mg} / \ell$ in groundwater samples from the Lower Shire Valley and up to $82 \mathrm{mg} / \ell$ in Nkhotakota have been reported (Bath, 1980). We recommend that future studies should include the levels of both dissolved (filtered) and total (including particulate) iron (and other metals). Total iron (and other metal) levels are in reality more representative of the aesthetic quality (colour and taste) of borehole water and its acceptability to water collectors and consumers.

In this study, all samples complied with the MBS MPL for magnesium; with concentrations on the east bank ranging from 11.4 to $51.3 \mathrm{mg} / \ell$ and on the west bank from 7.6 to $70.8 \mathrm{mg} / \ell$. Kempster et al. (1997) state that no undesirable health or aesthetic effects have been shown to be associated with magnesium concentrations in drinking-water of $<70 \mathrm{mg} / \ell$. These authors reported that levels $>30 \mathrm{mg} / \ell$ may impair the lathering of soap, and concentrations ranging from 70 to $100 \mathrm{mg} / \ell$ may impart a bitter taste to the water and cause diarrhoea in sensitive users, including infants. At levels above $100 \mathrm{mg} / \ell$ the bitter taste is exacerbated and diarrhoea may be expected, particularly in sensitive users.

Recent studies suggest that dissolved metals in drinkingwater such as magnesium and calcium may make a significant contribution to the dietary intake and reduce the risk of cardiovascular and osteoporosis diseases, respectively (WHO, 2009). In areas such as Chikhwawa, where many children and adults suffer from iron deficiency and anaemia, as a result of a poor diet, dietary intake of dissolved iron and other dissolved metals in drinking water may have similar positive health benefits.

Detectable levels of zinc, manganese and copper found in water samples were in general in compliance with the MBS MPL and in most cases with the WHO GV. In the few cases where the WHO GV was exceeded it was only marginally and at levels not thought to be harmful to health. Dissolved levels above $1 \mathrm{mg} / \ell$ copper, $0.3 \mathrm{mg} / \ell$ iron and $0.1 \mathrm{mg} / \ell$ manganese can give rise to aesthetic and organoleptic problems, associated with the staining of laundry, and impart a bitter taste to beverages (WHO, 2008). In this study, problems associated with the visual appearance of zigayigayi (particulates) floating in water and 'staining of white-coloured clothing' during washing were reported by water committees from a number of villages.

This problem is probably brought about by the oxidation of soluble metals, creating metal precipitates, by the mechanical agitation processes involved during water transportation, decantation into storage containers and hand-washing of clothes. Some water committee members stated that women used dish-cloths to filter out particulate material before storage in clay pots. A number of water committees also complained of a bitter and sharp taste to some borehole water supplies; however this information cannot be matched up with those water supplies that were found to slightly exceed the copper, iron, manganese and zinc levels highlighted above. The appearance of unsightly particulates in drinking-water and/or associated undesirable taste imparted to the water by other chemicals may result in consumers reverting back to unprotected sources, with the associated health, safety and welfare problems highlighted above. Bath in 1980 also observed that high iron levels (unfiltered) in water were one of the main reasons for well abandonment in Malawi.

Recorded chromium levels from boreholes located on both banks of the Shire River (max. $1.3 \mu \mathrm{g} / \ell$ ) were well below the MBS MPL and WHO health-based guideline value. These levels are in accordance with the levels expected in drinking water $(2 \mu \mathrm{g} / \ell)$ which is not subject to industrial pollution (WHO, 2008), and are similar to the concentrations detected in groundwater studies conducted in other parts of the world (WHO 2003a). The levels reported here are for total chromium and no attempt was made to differentiate between chromium III and the carcinogenic chromium VI.

The presence of arsenic, mercury, vanadium, selenium, nickel, lead, cadmium, aluminium and cobalt was not detected in borehole samples above minimum detection limits. 


\section{CONCLUSION}

This study presents data on the chemical quality of groundwater supplies in Chikhwawa, Malawi, and is the most comprehensive undertaken to date. In general, most parameters complied with the Malawi Bureau of Standards Maximum Permissible Levels (MBS MPL) for borehole water supplies. The MBS MPL standards for iron, sodium and nitrate were slightly exceeded at a few boreholes, technically rendering the water supply unwholesome but not necessarily unfit for human consumption. In contrast, significantly high nitrate $(\leq 200 \mathrm{mg} / \ell)$ and fluoride $(\leq 5 \mathrm{mg} / \ell)$ concentrations were detected in borehole samples in a number of villages and warrant further investigation. The borehole at Miagno is particularly worthy of further investigation due to the elevated levels of a range of water quality parameters. The highest nitrate concentration recorded was, on average, 4 times the MBS MPL and WHO GV. These concentrations present a significant medical risk (SMR) to the health of bottle-fed infants, when consumed over a relatively short period of time, especially in the presence of microbial contamination. Levels of fluoride detected were in accordance with those known to cause dental and skeletal fluorosis when consumed over a prolonged period of time, and hence also constitute a SMR risk to the health of the consumer.

Water committee members complained of problems associated with taste and appearance in borehole water supplies primarily on the west bank, presumably as a result of the high $\mathrm{Cl}$ levels and precipitation of soluble Fe and Mn. This resulted in some members within village communities reverting to the use of unprotected sources to obtain drinking-water; ideally this practice should be dissuaded through the education of water and village health committees. Surface water sources in Chikhwawa are highly polluted from a variety of point and non-point sources. It is recommended that further studies determine both total and dissolved iron and manganese levels, as these will be considerably higher in unfiltered samples and more reflective of the appearance and acceptability of the source for drinking-water purposes.

In many parts of the developing world there is increasing concern about contamination of groundwater from agricultural, industrial and municipal sources in addition to naturally occurring chemicals. Based on the findings of this study further research is required to determine the impact of agricultural sources of pollution, especially the sugarcane plantation industry, on borehole water supplies. Although the aim of this study was to determine the wholesomeness, acceptability and fitness of borehole-derived drinking water for human consumption, there is need for further study to determine the geological, hydrological and geo-chemical characteristics of the underground rock strata and aquifers in the area that affect water quality, over a prolonged period of time to include seasonal factors.

\section{ACKNOWLEDGEMENTS}

We would like to acknowledge the help and assistance of the late Mr J Gausi, Ministry of Irrigation and Water Development, Malawi. For assistance with the project and processing of samples we thank Mr A Maliro, Department of Applied Sciences, University of Malawi - The Polytechnic and $\mathrm{Mr}$ M Gibson, Department of Civil Engineering, University of Strathclyde.

\section{REFERENCES}

APHA (1998) Standard Methods for the Examination of Water and Wastewater $\left(20^{\text {th }}\right.$ edn.). American Public Health Association, Washington DC.

BATH AH (1980) Hydrochemistry in groundwater development: report on an advisory visit to Malawi. British Geological Survey Report, WD/OS/80/20. British Geological Survey, London.

BORDALO AA and SAVVA-BORDALO J (2007) The quest for safe drinking water: An example from Guinea-Bissau (West Africa). Water Res. 41 2978-2986.

CHILTON PJ AND SMITH-CARINGTON A (1984) Characteristics of the weathered basement aquifer in Malawi in relation to rural water supplies. In: Challenges in African Hydrology and Water Resources. IAHS Publication No. 144. International Association of Hydrological Sciences, Wallingford. 57-72.

EPA (ENVIRONMENTAL PROTECTION AGENCY, USA) (2001) National Primary Drinking Water Regulations, Environmental Protection Agency, United States of America, Washington. URL: http://www.epa.gov/safewater/dwh.

GRIMASON AM, BEATTIE TK, MORSE TD, MASANGWI SJ, JABU GC, TAULO SC and LUNGU KK (2013) Classification and quality of groundwater supplies in the Lower Shire Valley, Malawi. Part 2: Classification of borehole water supplies in Chikhwawa, Malawi. Water SA 39 (4) 573-582.

KANYERERE TOB, MACPHERSON GMN and MKANDAWIRE T (2010) Rural water supply and sanitation in Malawi: Groundwater context. In: Xu Y and Braune E (eds.) Sustainable Groundwater Resources in Africa. Water Supply and Sanitation Environment. CRC Press, Boca Raton. ISBN: 978-0-415-87603-2.

KEMPSTER PL, VAN VLIET HR and KUHN A (1997) The need for guidelines to bridge the gap between ideal drinking-water quality and that quality which is practically available and acceptable. Water SA 23 (2) 163-167.

LANGENEGGE O (1994) Groundwater Quality and Handpump Corrosion in Africa. Water and sanitation Report 8. UNDP-World Bank Water and Sanitation Program, Washington D.C.

MACFARLANE MJ and BOWDEN DJ (1992) Mobilization of aluminium in the weathering profiles of the African surface in Malawi. Earth Surf. Processes Landforms 17 789-805.

MBS (MALAWI BUREAU OF STANDARDS) (2005) Malawi Bureau of Standards Borehole and Shallow Well Water Quality Specification. MS 733:2005 $1^{\text {st }}$ Edition. Malawi Bureau of Standards, Blantrye.

MIWD (MINISTRY OF IRRIGATION AND WATER DEVELOPMENT, MALAWI) (2002) Development devolution of functions to assemblies: Guidelines and standards. Government of Malawi, Ministry of Irrigation and Water Development, Lilongwe.

NCHILIKISO E (2011) Personal communication, 15 October 2011. Mr. E. Nchilikiso, Chikhwawa District Officer, Tikwere House, Private Bag 390, Lilongwe, Malawi.

NSO (NATIONAL STATISTICAL OFFICE, MALAWI) (2005) Integrated Household Survey 2004-2005. National Statistical Office, Government of Malawi, Zomba.

PALAMULENI LG (2002) Effect of sanitation facilities, domestic solid waste disposal and hygiene practices on water quality in Malawi's urban poor areas: a case study of South Lunzu Township in the city of Blantyre. Phys. Chem. Earth 27 845-850.

SAJIDU SM, MASUMBA FFF, FABIANO E and NGONGONDO C (2007) Drinking water quality and identification of fluoritic areas in Machinga, Malawi. Malawi J. Sci. Technol. 8 42-56.

SAJIDU SM, MASAMBA WRL, THOLE B and MWATSETEZA JF (2008) Groundwater fluoride levels in villages of Southern Malawi and removal studies using bauxite. Int. J. Phys. Sci. 3 (1) 1-11.

UN (1989) Malawi. In: Groundwater in Eastern, Central and Southern Africa. Natural Resources/Water Series No. 19. United Nations, New York.

WHO (2003a) Chromium in Drinking-Water. Originally published in: Guidelines for Drinking-Water Quality (2 ${ }^{\text {nd }}$ edn.) Volume 2, Health Criteria and Other Supporting Information. World Health Organisation, Geneva 1996. 
WHO (2003b) Iron in Drinking-Water. Background document for development of WHO Guidelines for Drinking-Water Quality. Originally published in: Guidelines for Drinking-Water Quality ( $2^{\text {nd }}$ edn.) Volume 2, Health Criteria and Other Supporting Information. World Health Organisation, Geneva 1996.

WHO (2008) Guidelines for Drinking-Water Quality. (Third edition incorporating the first and second addenda). Volume 1 , Recommendations. World Health Organization, Geneva. ISBN 9789241547611
WHO (2009) The mineral composition of water and its contribution to calcium and magnesium intake. Chapter 3. In: Cutrovo J and Bartram J (eds.) Calcium and Magnesium in Drinking Water. Public Health Significance. 37-58. ISBN 9789241563550.

WHO (2010) Progress on Sanitation and Drinking-water: 2010 Update. WHO/UNICEF Joint Monitoring Programme for Water Supply and Sanitation. World Health Organization, Geneva. ISBN 9789241563956.

WHO (2011) Guidelines for Drinking-Water Quality (4 $4^{\text {th }}$ edn.). World Health Organization, Geneva. ISBN 9789241548151. 